\title{
Intentional marginal resection of periosteal osteosarcoma in combination with neoadjuvant chemotherapy: A report of two cases and a review of the literature
}

\author{
YU CHEN, SONG-FENG XU, MING XU and XIU-CHUN YU \\ Department of Orthopedic Surgery, General Hospital of Jinan Military Commanding Region, \\ Jinan, Shandong 250031, P.R. China
}

Received April 9, 2015; Accepted June 10, 2016

DOI: $10.3892 / \mathrm{ol} .2017 .5556$

\begin{abstract}
A wide resection of the tumor with or without chemotherapy is the treatment of choice for periosteal osteosarcoma (PO) of the extremities, however, post-operative limb function and quality of life could be compromised. The present study reports two cases of 14-year-old boys who presented with progressively enlarging masses in their right knee regions. Computed tomography and magnetic resonance imaging scans indicated a fusiform space-occupying mass encircling the bone cortex, with stippled calcification. A diagnosis of PO was suspected. The histological findings confirmed the diagnosis of intermediate PO. Pre-operative chemotherapies were started, and good responses were detected by clinical evaluation and histological findings. Surgeries preserving the functional structures, including neurovascular bundles, tendons, muscles and epiphyses, were performed, followed by routine chemotherapy. The two patients experienced disease-free survival with follow-up times of 37 and 108 months, respectively. The patients were satisfied with the results of the treatment and they returned to normal life activities. These two cases indicated that a marginal resection of the tumor in conjunction with effective neoadjuvant chemotherapy may be an ideal alternative treatment for intermediate PO, since survival along with well-preserved limb function could be guaranteed. By contrast, a wide excision could result in the loss of limb function.
\end{abstract}

\section{Introduction}

Periosteal osteosarcoma (PO) is a rare tumor, which usually originates from the diaphysis of long bones (1). On radiological

Correspondence to: Professor Xiu-Chun Yu, Department of Orthopaedic Surgery, General Hospital of Jinan Military Commanding Region, 25 Shifan Road, Jinan, Shandong 250031, P.R. China

E-mail: toeflmugou@163.com

Key words: periosteal osteosarcoma, neoadjuvant chemotherapy, marginal resection examination, PO presents as fusiform masses located in the periosteum that are accompanied by periosteal reactions and a soft tissue mass (1). PO represents $<2 \%$ of all osteosarcomas (1) and this low incidence is mirrored by the lack of large case series. The diagnosis of PO should preclude high-grade surface sarcoma and parosteal sarcoma $(2,3)$. Compared with other osteosarcoma subtypes, PO has a low propensity to metastasize, and it is histologically an intermediate-grade tumor. The therapeutic regimen for PO is controversial (4-6), although there is a widely accepted consensus that a wide surgical excision is the mainstay of treatment for PO (3-6). However, post-operative limb function is usually compromised by a wide excision as the procedure results in a considerable wound, which leads to difficulties regarding reconstruction and rehabilitation $(5,6)$. In the present study, a marginal resection was manipulated in the setting of pre-operative chemotherapy for two patients with intermediate PO; post-operative limb function was well preserved and the patients were satisfied with the therapeutic outcome. The current study presents the detailed therapeutic strategies used for these two patients and conducts a review of the literature. Written informed consent was obtained from the patients' families in both cases.

\section{Case report}

Case 1. A 14-year-old boy presented to the General Hospital of Jinan Military Commanding Region (Jinan, Shandong, China) in July 2010 with a 4-week history of a progressively enlarging mass and pain in the right knee. On examination, a tender, firm lump was apparent in the right medial knee. The patient experienced difficulty in walking, with fixed right knee flexion of $40^{\circ}$. Imaging examinations, including X-ray, computed tomography (CT) and magnetic resonance imaging (MRI) showed an osseous fusiform mass encircling the right distal femur, whilst the periosteum was intact (Fig. 1A-C). Tissue samples were paraffin-embedded, fixed and cut into 5- $\mu \mathrm{m}$ sections. The tissue sections were fixed with formalin. A biopsy of the lesion confirmed a diagnosis of PO; the hematoxylin and eosin-stained slides showed spindle cells with osteoid matrix, indicating an intermediate-grade tumor (Fig. 2A), according to the grading system described by Unni and Dahlin (7). Bone scans and CT of the thorax ruled out metastasis. The patient 

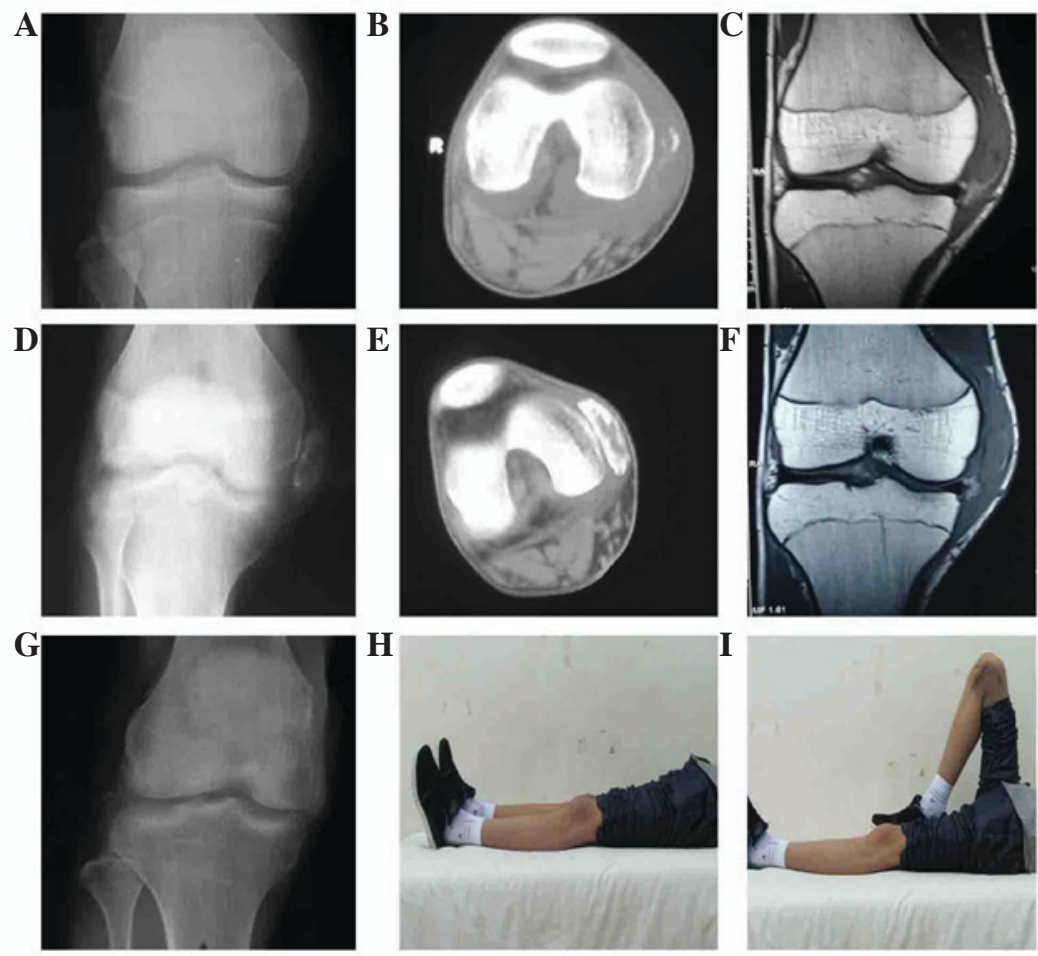

Figure 1. Case 1: Imaging examinations, including (A, D and G) X-ray, (B and E) computed tomography and (C and E) magnetic resonance imaging (MRI) (A-C) A mass lesion encircling the cortex of distal femur, with no intramedullary involvement. (D-F) Radiological changes owing to pre-operative chemotherapy; diminishing swelling, a well-defined mass and calcification were detected. MRI indicated a smaller volume of tumor. (G) Radiological finding at the end of 37 months of follow-up showing no local recurrence. (H and I) Well preserved limb function, as demonstrated by normal (H) flexion and (I) extension, at the end of the follow-up period.

A

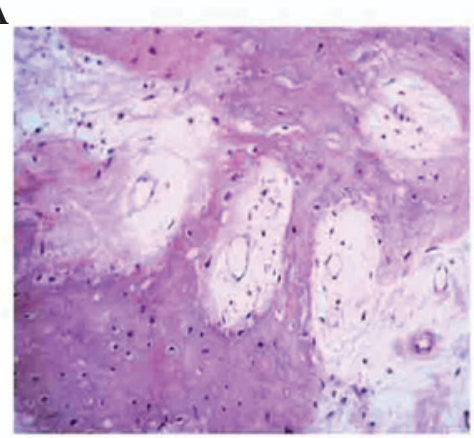

B

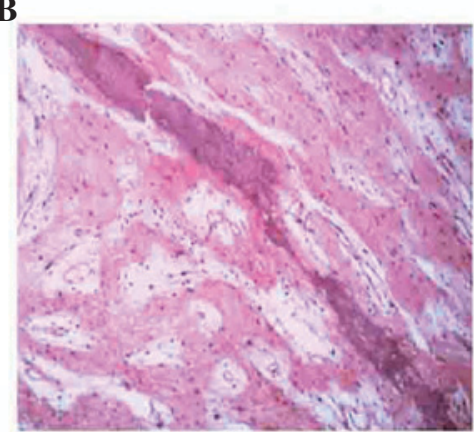

Figure 2. Histopathological findings of (A) case 1 and (B) case 2 showing characteristic cells with an osteoid matrix, indicating an intermediate-grade periosteal osteosarcoma (hematoxylin and eosin stain; magnification, x100).

was started on two 2-week cycles of chemotherapy $\left(8-12 \mathrm{~g} / \mathrm{m}^{2}\right.$ high-dose methotrexate, $30 \mathrm{mg} / \mathrm{m}^{2}$ doxorubicin and $2.0 \mathrm{~g} / \mathrm{m}^{2}$ ifosfamide) with an interval of 2 weeks, and experienced pain relief, shrinkage of the mass and an improvement in right knee motion $\left(0-75^{\circ}\right)$. Furthermore, imaging findings showed that the mass had become well-defined, with peripheral calcification (Fig. 1D-F). Surgery was performed 2 weeks after the chemotherapy in the form of an excision of the tumor, preserving the main structures, including the neurovascular bundles, tendons, muscles and epiphyses. A limited excision of the peripheral soft tissue $(<2 \mathrm{~cm}$ from the tumor) and bone $(<3 \mathrm{~cm}$ from the tumor) was performed, and biological reconstruction of the right medial collateral ligament was performed by forward locating the right semitendinosus muscle tendon. The post-operative histological findings indicated a good response to the pre-operative chemotherapy (necrosis percentage, $\geq 90 \%$ ) and a negative excision margin. A single 2-week cycle of chemotherapy $\left(8-12 \mathrm{~g} / \mathrm{m}^{2}\right.$ high-dose methotrexate, $30 \mathrm{mg} / \mathrm{m}^{2}$ doxorubicin and $2.0 \mathrm{~g} / \mathrm{m}^{2}$ ifosfamide), was performed at 2 weeks post-surgery, with an interval of 2 weeks. After being discharged, the patient received five 2 -week cycles of chemotherapy $\left(8-12 \mathrm{~g} / \mathrm{m}^{2}\right.$ high-dose methotrexate, $30 \mathrm{mg} / \mathrm{m}^{2}$ doxorubicin and $2.0 \mathrm{~g} / \mathrm{m}^{2}$ ifosfamide), with an interval of 2 weeks between chemotherapy cycles. At the end of the follow-up period, the range of motion in the right knee was normal $\left(0-130^{\circ}\right)$. Radiological findings demonstrated 

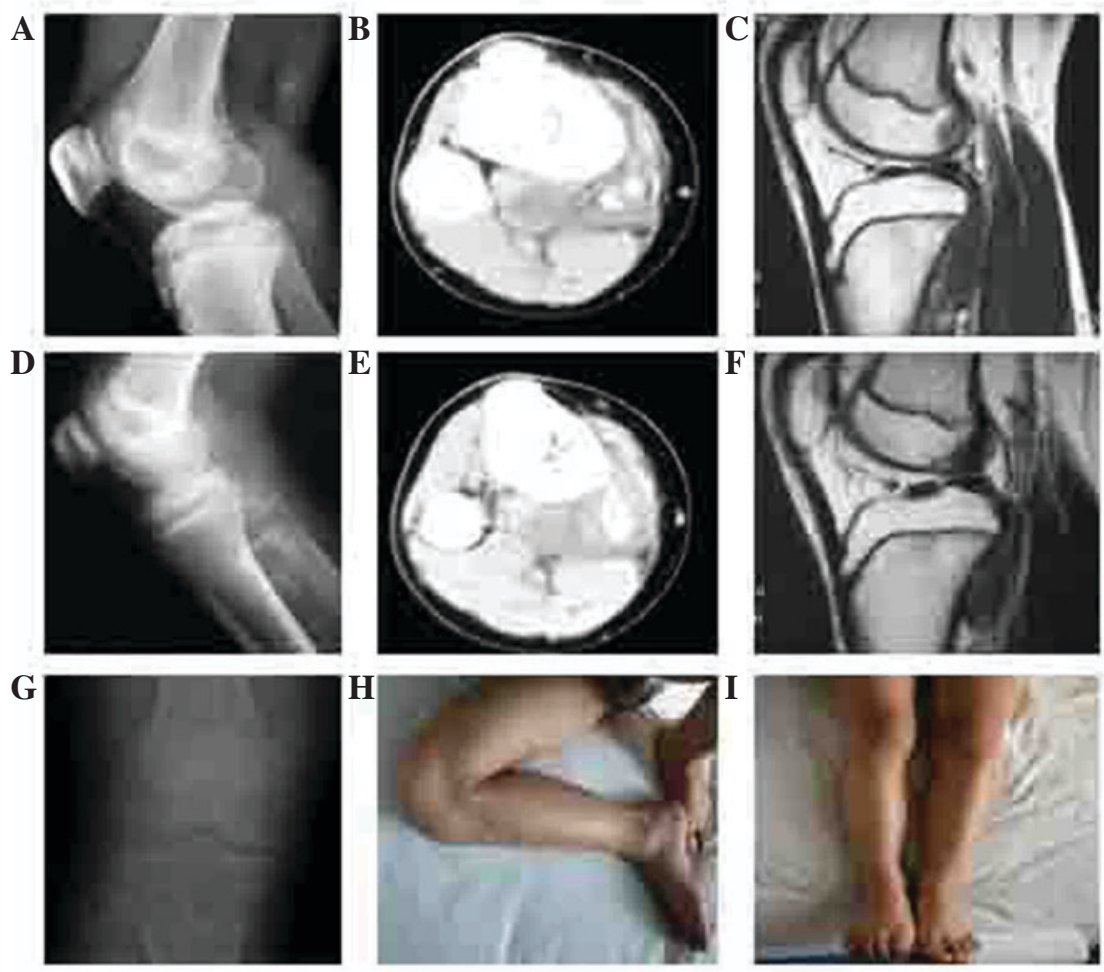

Figure 3. Case 2: Imaging examinations, including (A, D and G) X-ray, (B and E) computed tomography and (C and E) magnetic resonance imaging (MRI). (A-C) A space-occupying lesion in the proximal region of the right tibia, with no intramedullary involvement. (D-F) Radiological changes owing to pre-operative chemotherapy; the mass was well-defined and calcification was detected within it. MRI indicated a smaller volume of tumor. (G) X-ray at the end of 108 months of follow-up showing no local recurrence. (H and I) Normal limb function, as demonstrated by as demonstrated by normal (H) flexion and (I) extension, at the end of the follow-up period.

no metastases or recurrence (Fig. 1G). Normal life activities were recorded until the end of the follow-up time of 37 months (Fig. 1H and I). According to the Musculo-Skeletal Tumor Society (MSTS) functional scoring system, the patient's score was $100 \%$ (8).

Case 2. A 14-year-old boy was referred to the General Hospital of Jinan Military Commanding Region in August 2004 due to a suspicious lesion in the right proximal medial tibia. Clinical examination revealed a tender, bony-hard lump on the proximal tibia. The patient's ambulatory ability was constrained, with fixed right knee flexion of $30^{\circ}$. Imaging findings indicated a space-occupying mass adjacent to the cortex of the right proximal tibia (Fig. 3A-C). Tissue samples were paraffinembedded, fixed and cut into 5- $\mu \mathrm{m}$ sections for staining. The tissue sections were fixed with formalin. A diagnosis of PO was determined by histopathological results; hematoxylin and eosin-stained slides showed spindle cells with an osteoid matrix, indicating that the tumor was of intermediate grade (Fig. 2B), according to the grading system described by Meister et al (9). As a routine process, metastases was ruled out. Next, two 2 -week cycles of chemotherapy $\left(120 \mathrm{mg} / \mathrm{m}^{2}\right.$ cisplatin, $30 \mathrm{mg} / \mathrm{m}^{2}$ doxorubicin and $2.0 \mathrm{~g} / \mathrm{m}^{2}$ ifosfamide) with an interval of 2 weeks were started. The patient's symptoms were relieved along with improving imaging findings, including a well-defined tumor border and progressive calcification (Fig. 3D-F). A portion of the local tibial cortex and peripheral soft tissue were removed along with the tumor in accordance with the criteria for a marginal excision. The main functional structures were preserved. The excised bone was soaked in $99 \%$ alcohol for inactivation, followed by replantation. No other biological reconstruction was performed. The post-operative histological findings determined a good response and a negative margin. Routine chemotherapy (2-week cycle of $120 \mathrm{mg} / \mathrm{m}^{2}$ cisplatin, $30 \mathrm{mg} / \mathrm{m}^{2}$ doxorubicin and $2.0 \mathrm{~g} / \mathrm{m}^{2}$ ifosfamide with an interval of 2 weeks) was commenced at 2 weeks post-surgery. After being discharged, the patient underwent another six 2-week cycles of chemotherapy $\left(120 \mathrm{mg} / \mathrm{m}^{2}\right.$ cisplatin, $30 \mathrm{mg} / \mathrm{m}^{2}$ doxorubicin and $2.0 \mathrm{~g} / \mathrm{m}^{2}$ ifosfamide) with an interval of 2 weeks. The patient experienced disease-free survival in 108 months of follow-up (Fig. 3G). The range of motion in the right knee was normal $\left(0-130^{\circ}\right.$; Fig. $3 \mathrm{H}$ and $\left.\mathrm{I}\right)$, and the patient's MSTS score was $100 \%$. The patient had recommenced normal life activities during the follow-up period.

\section{Discussion}

Previous studies have demonstrated that $\mathrm{PO}$ has less aggressive behavior compared with other osteosarcoma subtypes (10). According to one of these studies, a PO diagnosis should preclude intramedullary involvement, which may suggest tumor progression (3). By contrast, opposing studies have concluded that medullary extension is frequent among PO cases (11). Gulia et al (12) suggested that intramedullary involvement may suggest the aggressive nature of PO. The present study did not include medullary extension, however, we hypothesize that if patients with intramedullary involvement 
were included, the tumor grades could be elevated, resulting in adverse impact on the results.

Since being introduced, multiple-agent chemotherapy has greatly improved the long-term survival rate among osteosarcoma patients, which is now $49-76 \%$ (13). The frontline chemotherapeutic agents for osteosarcoma include methotrexate, cisplatin, doxorubicin and ifosfamide (14). However, the role of chemotherapy as a treatment for PO has been doubted. Since PO has less aggressive behavior than other sarcoma subtypes, chemotherapy may be absent in intermediate-grade PO (1,3-6). Clinical studies have demonstrated that local recurrence rather than chemotherapy is an important prognostic factor for survival in PO $(6,15)$. A single center study indicated that wide resection is the only predictor for survival (1). However, a review of 17 cases with a mean follow-up time of 52 months showed the encouraging effects of chemotherapy, with results indicating $100 \%$ metastasis-free survival among PO cases (3). Furthermore, the outcome indicated that it may be too prudent for the decision to use chemotherapy to be only shared with patients with high-grade PO, suggesting that chemotherapy may be routinely utilized in PO of intermediate grade $(1,3,16)$. In the present study, chemotherapy was administered in two intermediate-grade PO patients who proved to be good responders to the treatment, and who experienced disease-free survival times of 37 and 108 months, respectively, at last follow-up. Based on these findings, chemotherapy may be beneficial to patients with PO of intermediate grade.

There is wide consensus regarding aggressive resection being used for PO $(1,17,18)$. Tumors have been reported to spread further than could be imaged using CT or MRI, and in addition, an incomplete tumor resection is considered as the most important risk factor for an adverse outcome. Therefore, a wide excision is considered mandatory to achieve the complete removal of a tumor $(1,19,20)$. However, a wide excision often necessitates removal of functional structures, resulting in prolonged surgery time and compromised limb function (3,8,20-22). Furthermore, recurrences and metastases are not rare in patients undergoing a wide excision of a tumor $(1,19)$. Therefore, in the present study, marginal excision of tumor preserving functional structures was sought in the setting of effective neoadjuvant chemotherapy; two cases without intramedullary involvement were enrolled to ensure the intermediate tumor grade of the patients. The survival outcome was encouraging, but more importantly, the patients returned to normal daily activities and were satisfied with the outcome of the treatment (22-24).

It is noteworthy that the discrimination of good responders to chemotherapy is crucial for marginal excision manipulation. Sumiya et al (25) argued that when a good response was documented by at least two out of four radiological modalities, including X ray, MRI, angiography and thallium-201 scintigraphy, $>90 \%$ tumor necrosis could be expected. In the present study, a good response originated from symptomatic relief, improved imaging findings and a good histopathological response to chemotherapy. It should also be highlighted that only PO cases of intermediate grade were included, and that further studies are warranted to verify the effectiveness of marginal excision along with chemotherapy for patients with higher grade PO.
In conclusion, marginal surgical removal of the tumor in conjunction with effective neoadjuvant chemotherapy may improve survival in patients with intermediate PO. Furthermore, limb function could be restored to a good standard. The discrimination of good responses to chemotherapy could be important for marginal excision manipulation.

\section{References}

1. Cesari M, Alberghini M, Vanel D, Palmerini E, Staals EL, Longhi A, Abate M, Ferrari C, Balladelli A and Ferrari S: Periosteal osteosarcoma: A single-institution experience. Cancer 117: 1731-1735, 2011.

2. Schajowicz F, McGuire MH, Santini Araujo E, Muscolo DL and Gitelis S: Osteosarcomas arising on the surfaces of long bones. J Bone Joint Surg Am 70, 555-564, 1988.

3. Revell MP, Deshmukh N, Grimer RJ, Carter SR and Tillman RM: Periosteal osteosarcoma: A review of 17 cases with mean follow-up of 52 months. Sarcoma 6: 123-130, 2002.

4. Okada K, Unni KK, Swee RG and Sim FH: High grade surface osteosarcoma: A clinicopathologic study of 46 cases. Cancer 85: 1044-1054, 1999.

5. Murphey MD, Jelinek JS, Temple HT, Flemming DJ and Gannon FH: Imaging of periosteal osteosarcoma: Radiologic-pathologic comparison. Radiology 233, 129-138, 2004.

6. Grimer RJ, Bielack S, Flege S, Cannon SR, Foleras G, Andreeff I, Sokolov T, Taminiau A, Dominkus M, San-Julian M, et al: Periosteal osteosarcoma-a European review of outcome. Eur J Cancer 41: 2806-2811, 2005.

7. Unni KK and Dahlin DC: Grading of bone tumours. Semin Diag Pathol 1: 165-172, 1984

8. Kanazawa Y, Tsuchiya H, Nonomura A, Takazawa K, Yamamoto $\mathrm{N}$ and Tomita $\mathrm{K}$ : Intentional marginal excision of osteosarcoma of the proximal fibula to preserve limb function. J Orthop Sci 8, 757-761, 2003.

9. Meister P, Konrad E, Lob G, Janka G, Keyl W and Stürz H: Osteosarcoma: Histological evaluation and grading. Arch Orthop Trauma Surg 94: 91-98, 1979.

10. Ma Q, Zhou Y, Ma B, Chen X, Wen Y, Liu Y, Fan Q and Qiu X: The clinical value of CXCR4, HER2 and CD44 in human osteosarcoma: A pilot study. Oncol Lett 3: 797-801, 2012.

11. Hall RB, Robinson LH, Malawar MM and Dunham WK: Periosteal osteosarcoma. Cancer 55: 165-171, 1985.

12. Gulia A, Puri A, Pruthi M and Desai S: Oncological and functional outcome of periosteal osteosarcoma. Indian J Orthop 48: 279-284, 2014.

13. Saeter G, Alvegård TA, Elomaa I, Stenwig AE, Holmström T and Solheim OP: Treatment of osteosarcoma of the extremities with the T-10 protocol, with emphasis on the effects of preoperative chemotherapy with single-agent high-dose methotrexate: A Scandinavian sarcoma group study. J Clin Oncol 9: 1766-1775, 1991.

14. Arpaci F, Ataergin S, Ozet A, Erler K, Basbozkurt M, Ozcan A, Komurcu S, Ozturk B, Celasun B, Kilic S and Kuzhan O: The feasibility of neoadjuvant high-dose chemotherapy and autologous peripheral blood stem cell transplantation in patients with nonmetastatic high grade localized osteosarcoma: Results of a phase II study. Cancer 104: 1058-1065, 2005.

15. Rose PS, Dickey ID, Wenger DE, Unni KK and Sim FH: Periosteal osteosarcoma: Long-term outcome and risk of late recurrence. Clin Orthop Relat Res 453 314-317, 2006.

16. Papagelopoulos PJ, Galanis E, Sim FH and Unni KK: Periosteal osteosarcoma. Orthopedics 22 971-974, 1999.

17. Singh D, Sen R, Chaudhary S and Tripathy SK: Periosteal osteosarcoma of the calcaneum: A case report. Foot Ankle Spec 5: 121-123, 2012.

18. Maheshwari AV, Jelinek JS, Seibel NL, Meloni-Ehrig AM, Kumar D and Henshaw RM: Bilateral synchronous tibial periosteal osteosarcoma with familial incidence. Skeletal Radiol 41: 1005-1009, 2012.

19. Masterson EL, Ferracini R, Davis AM, Wunder JS and Bell RS: The geometric osteotomy: Joint preservation in juxta-articular surface bone neoplasms. Sarcoma 1: 167-174, 1997.

20. Tsuchiya H, Tomita K, Mori Y, Asada N and Yamamoto N: Marginal excision for osteosarcoma with caffeine assisted chemotherapy. Clin Orthop Relat Res: 27-35, 1999. 
21. Kabukcuoglu Y, Grimer RJ, Tillman RM and Carter SR: Endoprosthetic replacement for primary malignant tumors of the proximal femur. Clin Orthop Relat Res: 8-14, 1999.

22. Tsuchiya H, Tomita K, Mori Y, Asada N, Morinaga T, Kitano S and Yamamoto N: Caffeine-assisted chemotherapy and minimized tumor excision for nonmetastatic osteosarcoma. Anticancer Res 18: 657-666, 1998.

23. Enneking WF, Dunham W, Gebhardt MC, Malawar M and Pritchard DJ: A system for the functional evaluation of reconstructive procedures after surgical treatment of tumors of the musculoskeletal system. Clin Orthop Relat Res: 241-246, 1993.
24. Winkler K, Beron G, Delling G, Heise U, Kabisch H, Purfürst C, Berger J, Ritter J, Jürgens H, Gerein V, et al: Neoadjuvant chemotherapy of osteosarcoma: Results of a randomized cooperative trial (COSS-82) with salvage chemotherapy based on histological tumor response. J Clin Oncol 6: 329-337, 1988.

25. Sumiya H, Taki J, Tsuchiya H, Nonomura A, Miyauchi $\mathrm{T}$ and Tonami N: Midcourse thallium-201 scintigraphy to predict tumor response in bone and soft-tissue tumors. J Nucl Med 39: 1600-1604, 1998. 